This is a postprint version of the following published document:

Jerez, B., et al. Flexible electro-optic, single-crystal difference frequency generation architecture for ultrafast mid-infrared dual-comb spectroscopy. In: ACS Photonics, 5(6), June 2018, Pp. 2348-2353

DOI: https://doi.org/10.1021/acsphotonics.8b00143

(C) 2018 American Chemical Society 


\title{
Flexible Electro-Optic, Single-Crystal Difference Frequency Generation Architecture for Ultrafast Mid-Infrared Dual-Comb Spectroscopy
}

\author{
Borja Jerez, ${ }^{* \odot}$ Pedro Martín-Mateos, ${ }^{\odot}$ Frederik Walla, Cristina de Dios, and Pablo Acedo \\ Electronics Technology Department, Universidad Carlos III de Madrid, C/Butarque 15, 28911 Leganés, Madrid, Spain
}

ABSTRACT: Crowded with the fundamental signatures of many popular molecules, the mid infrared range of the electromagnetic spectrum is particularly attractive for applications ranging from identification of transient phenomena to sensing of trace gases. Dual comb spectroscopy is a technique that unveils the potential to access this region with a pair of phase locked optical frequency combs on a high resolution, real time basis without the mechanical limitations of traditional spectrometers. As the ideal

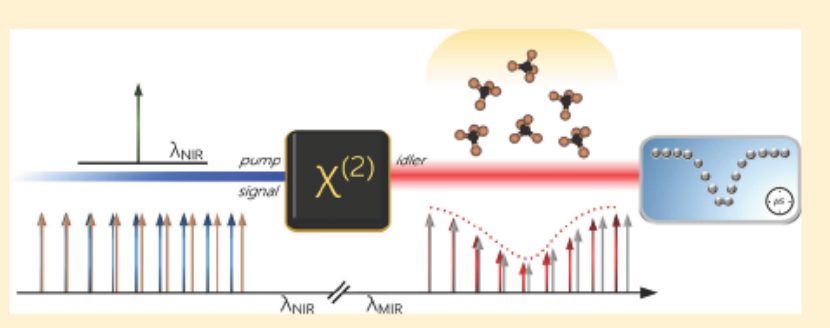
characteristics of an optical frequency comb are strongly influenced by the target application, electro optic dual comb systems are one of the most promising solutions with full capabilities to neatly fit to the application of interest beyond laboratory environments. Parameters such as resolution, measurement speed, or central wavelength are easily adjustable by means of compact, low cost arrangements based on commercial off the shelf components. To fully exploit their potential for molecular spectroscopy, we present here a modular instrument designed to perform ultrafast dual comb spectroscopy in the mid infrared region. The architecture comprises a fiberized near infrared electro optic dual comb scheme and a single crystal difference frequency generation module to generate mid infrared combs, thus significantly alleviating the complexity of the free space setup while preserving absolute independence between the instrument and the sample of study. The feasibility of the instrument is successfully validated by recovering the absorption profile of methane at $2896.98 \mathrm{~cm}^{-1}$ within tens of microseconds.

KEYWORDS: mid infrared dual comb spectroscopy, difference frequency generation, electro optic optical frequency combs, optical gas analysis, absorption spectroscopy, real time measurements, multiheterodyne detection, MgO:PPLN crystals

$\mathrm{O}$ ptical frequency combs (OFCs) are ensembles of evenly spaced, tightly phase locked spectral lines in the frequency domain, in a similar fashion to a collection of harmonized lasers. ${ }^{1}$ The picture of this Nobel winning technology closely resembles that of an ultraprecise frequency ruler that enables the measurement and control of the light in a parallel way, and its potential was shortly devised for a number of applications, including molecular spectroscopy of gas phase species. ${ }^{2}$ Among the range of existing methods to retrieve the spectral information associated with each of the lines of an OFC, dual comb spectroscopy $(\mathrm{DCS})^{3}$ is one of the most spellbinding methods since its early demonstrations in the past decade. ${ }^{4}$ The basics of this procedure stem from employing a second comb with slightly different line spacing to scan the probe comb and downconvert it to the radiofrequency (RF) domain. In this way, the discrete structure of the comb can be simultaneously accessed without resorting to slow frequency tuning or mechanical parts, as in Fourier transform infrared spectrometers, enabling high reso lution, time resolved measurements in any spectral region where an OFC can be generated.

If we focus on gas analysis, this set of virtues is particularly attractive in the mid infrared (MIR) spectral region, ${ }^{5}$ which is a favored region densely populated with the fundamental absorption bands of many molecules, allowing for ultrasensitive molecular spectroscopy. ${ }^{6}$ However, the potential of DCS in the
MIR has been hindered by the complexity of generating pairs of combs with high mutual coherence ${ }^{7,8}$ in this frequency region, a major requirement to take full advantage of DCS in terms of resolution and sensitivity. Additionally, field detection of trace gases demands compact, versatile, and easy to handle instru ments that can be operated not only in laboratory environments but also under unfavorable conditions. Clearly, these factorshighly coherent MIR combs and field capabilities-comprise a binomial that must be fulfilled by any gas sensing instrument to be deployed in real world applications.

Direct access to the MIR region with OFCs-and retrieval of information via coherent DCS-has been demonstrated with solid state mode locked lasers (MLLs). ${ }^{9}$ Nevertheless, MLL based DCS possesses two inherent drawbacks: a comb spacing defined by the physical length of the device and the need for bulky setups with complicated stabilization or data processing mechanisms. It is noteworthy, however, that efforts are being made to readapt these setups to field applications, ${ }^{10}$ which so far have been validated in the near infrared (NIR). ${ }^{11-13}$ Addition ally, promising compact alternatives to MIR DCS based on quantum/interband cascade lasers ${ }^{14-16}$ and microresonators (Kerr combs) ${ }^{17}$ have also been recently demonstrated. None 
theless, these approaches are still in their early stages and usually rely on tailored designs with inherent limitations in terms of optical resolution and tuning capabilities. On the other hand, a different, well established method consists of using the mature technology extensively developed in NIR combs in conjunction with nonlinear techniques to transfer radiation to MIR by means of optical parametric oscillators (OPOs) $)^{18-20}$ or difference frequency generation (DFG). ${ }^{21-23}$ Specifically, when either the MIR generated power or the bandwidth does not play a fundamental role for the application, DFG based systems are particularly well suited to readily transfer NIR radiation to the MIR domain in a single pass arrangement with the sole requirement of satisfying the phase matching condition in the nonlinear media that outputs the MIR radiation. ${ }^{24}$

As an alternative to seed MLLs in the NIR, ${ }^{25-27}$ electro optic (EO) DCS rapidly emerged as an appealing method for dual comb generation. ${ }^{28-30}$ EO DCS architectures are generated by strongly modulating a continuous wave $(\mathrm{CW})$ laser with a pair of electro optic modulators (EOMs). The leading element that illustrates the success of these schemes is $\mathbf{3}$ fold: the intrinsic mutual coherence between combs, since they are generated from the same seed laser; the unabridged, flexible control over the modulation frequency, which ultimately defines not only the spectral resolution but also the measurement speed, spectral coverage, and compression factor; and the possibility of using commercially accessible components for uncomplicated external customization. It is important to remark that standard EO DCS architectures cannot contend with MLLs concerning the number of spectral points. However, as most practical applications do not require octave spanning bandwidths to meet their sensing requirements, this apparent limitation can indeed be used to increase not only the sensitivity but also the measurement speed, an interesting feature that may be cleverly exploited by ultrafast sensing applications targeting fast chemical kinetics ${ }^{31}$ or single molecular transitions ${ }^{32}$ without any negative impact on the resolution.

In this article, we present a compact, modular instrument devised to perform MIR DCS in real time based on EO combs and DFG using a magnesium doped periodically poled lithium niobate (MgO:PPLN) crystal as nonlinear media. The system is essentially fiberized and utilizes only one single crystal to perform the nonlinear conversion, thus notably reducing the complexity and the alignment requirements of the system, while maintaining full independence over the target sample optical length. The flexible nature of the architecture allows for the optimization of the acquisition speed for a given resolution with tuning capabilities across the transparency range of the crystal (2.6$5.2 \mu \mathrm{m})$. As a validation, when targeting the spectral feature of $\mathrm{CH}_{4}$ at $2896.98 \mathrm{~cm}{ }^{1}$, its narrow absorption profile is recovered within microseconds with an optical resolution of $0.005 \mathrm{~cm}^{1}$.In this context, the estimated normalized limit of detection of the instrument is at the level of the parts per million.

\section{METHODS}

Principles of Electro-Optic Dual-Comb Spectroscopy. As stated in the introduction, electro optic dual comb architectures are built upon the modulation of a $\mathrm{CW}$ laser through a pair of EOMs in order to generate a limited number of sidebands with slightly different mismatch in their spacing. Although the concept is simple, there are some considerations and restrictions that should be borne in mind.

In the frequency domain (see Figure 1(a)), two OFCs with repetition frequencies $f_{\mathrm{RPP}}$ and $f_{\mathrm{RPP}}+\Delta$ are generated in each of
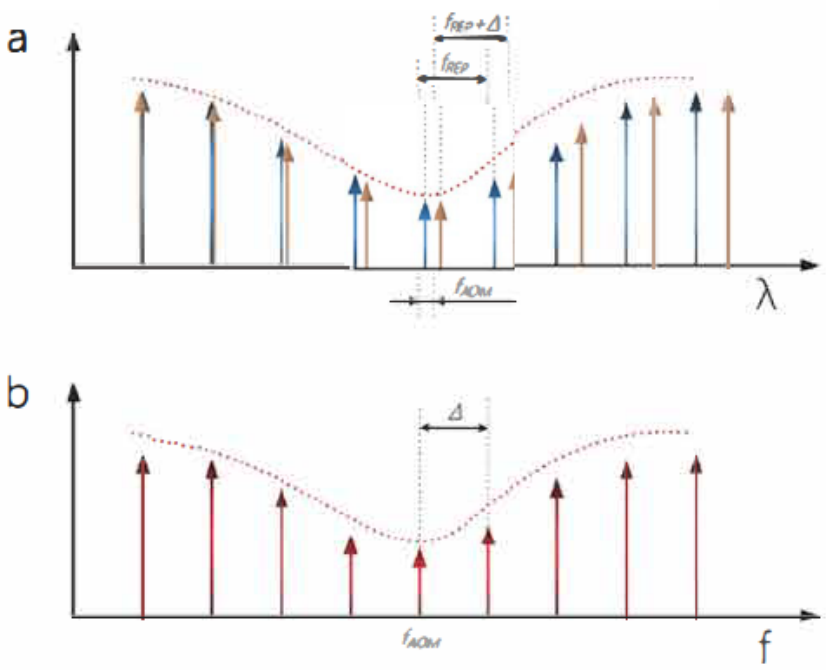

Figure 1. Principle of operation of EO DCS. Two combined, frequency shifted $f_{\text {AOM }}$ frequency combs with different repetition frequencies $f_{\text {REP }}$ and $f_{\text {REP }}+\Delta$ create a new RF comb with line spacing $\Delta$ after photodetection. The optical information engraved by, for example, an absorption feature is directly transferred to the RF domain, where it can be promptly accessed by the vast number of existing took and techniques. The passive mutual coherence of EO DCS systems allows for the unique mapping of each optical tooth into the RF domain, thus enabling high resolution measurements.

the arms of an interferometer that share a common light source (most commonly, a CW monochromatic laser). Additionally, one of the combs is frequency shifted by means of an AOM by a frequency $f_{\mathrm{AOM}}$. The combination and photodetection of these frequency combs give rise to a new comb in the RF domain (RF comb, Figure 1(b)) compressed by a factor of $f_{\mathrm{REP}} / \Delta$, whose offset frequency is defined by $f_{A O M}$ and the repetition frequency by $\Delta$. Since the RF comb mirrors the impact of external agents on the OFCs, any variation in the OFCs due to the absorption of light will be reflected in the RF comb. In this sense, the absorption caused by any gas species in the discrete comb structure can, therefore, be processed in the RF domain with a resolution ultimately limited by the linewidth of each OFC tooth.

It is important to note the value of $\Delta$ plays a major role in this technique. As the optical bandwidth of the comb grows, the pairs of lines that create the downconverted RF comb are farther between themselves, and eventually, one line from one comb may overlap with two lines of its counterpart, leading to an ambiguous mapping and the eventual loss of information. Analogously, the time domain picture is also important to understand this limitation. Here, each comb can be considered as a pulse train with slightly different time delay. When both pulse trains impinge on a photodetector, an interference pattern (usually denoted as interferogram) arises, which, once Fourier transformed, generates the above mentioned RF comb. The elapsed time until one interferogram fully crystallizes is defined bythe inverse ofthe difference between repetition rates, i.e., $1 / \Delta$.

Therefore, dual comb spectroscopy features an evident trade off between spectral coverage and speed of operation. Due to the moderate number of lines generated, EO dual comb architec tures favor the latter, which can be readily tuned with an RF synthesizer. Broader optical combs for DCS can be conveniently attained by means of a variety of different techniques; ${ }^{33-36}$ nevertheless, in order to avoid ambiguous mapping at fast measurement rates, these demonstrations need to resort to either filtering and stitching portions of the original spectrum (thus 


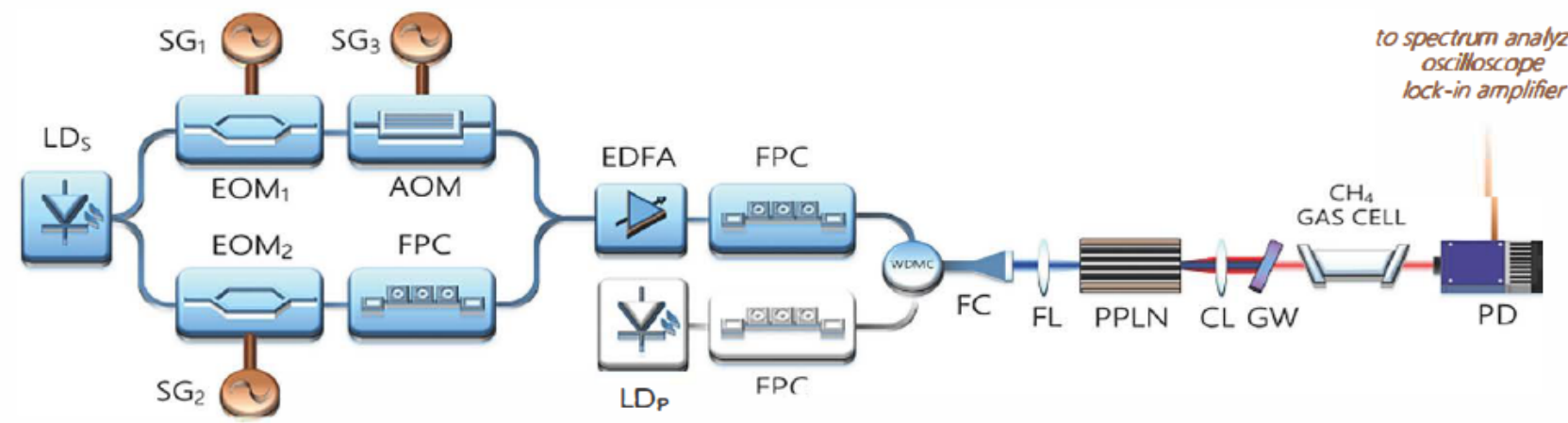

NIR ELECTRO OPTIC DUAL COMB

DFG MODULE

Figure 2. Schematic of the experimental setup. In alphabetical order: AOM, acousto optic modulator; CL, collimating lens; EDFA, erbium doped fiber amplifier; EOM, electro optic modulator; FC, fiber collimator; FI, focusing lens; FPC, fiber polarization controller; GW, germanium window; LD S/P, laser diode signal/pump; PD, photodetector; PPLN:, periodically poled lithium niobate crystal; SG, signal generator.

sacrificing the potential for single shot acquisition of the whole bandwidth $)^{21,24}$ or simply discarding a huge portion of the immense original spectrum, as in previously demonstrated field applications with MLLs. ${ }^{11,12}$ Notwithstanding these restrictions, it must be remarked that the appealing capabilities of dual comb schemes in terms of platform independent frequency resolution and measurement speed (as opposed to their FTIR or VIPA spectrometer counterparts) have confirmed them as one of the preferred tools for molecular spectroscopy among the scientific community.

Description of the Instrument. The experimental setup is depicted in Figure 2. The instrument presents a modular design, encompassing two autonomous units entirely built by commercial off the shelf components. First, an EO dual comb generation system is implemented through EO modulation of a CW laser. The seed laser is a Discrete Mode laser (Eblana Photonics, Inc.) emitting at $1538.63 \mathrm{~nm}$ with a tuning range of 4 $\mathrm{nm}$, whereas the EO combs (hereinafter referred as signal combs) are generated by means of two phase modulators (EOSpace, Inc.) with a $V_{n}$ of $3.3 \mathrm{~V}$. This two modulator configuration enables the number of generated teeth and their power distribution to be dynamically controlled through the algorithm presented elsewhere. ${ }^{37}$ In this case, a minimum of 50 adjacent lines within a $20 \mathrm{~dB}$ bandwidth is addressed at EOM modulation frequencies $\left(f_{\mathrm{REP}}\right)$ of $151 \mathrm{MHz}$ (which translates into an optical resolution of $0.005 \mathrm{~cm}^{1}$ ) with a frequency detuning $(\Delta)$ of 100 $\mathrm{kHz}$. Both twin EOMs are fed with RF signals previously amplified with commercial off the shelf RF amplifiers. The comb with line spacing $f_{\mathrm{REP}}+\Delta$ is further frequency shifted $f_{\mathrm{AOM}}$ by means of an acousto optic modulator (Gooch \& Housego, Inc.) driven at $82.5 \mathrm{MHz}$ with an RF input power of $24 \mathrm{dBm}$. Both combs are then recombined. A small fraction of the output power is used to monitor both generated RF comb (after photo detection in an InGaAs detector) and emission wavelength with a spectrum analyzer with $1 \mathrm{ppm}$ frequency accuracy and $20 \mathrm{pm}$ resolution. The resultant RF comb generated is shown in Figure 3. More than 60 clearly resolved beatnotes are generated (with 50 of them within the $20 \mathrm{~dB}$ bandwidth) in the downconversion process with a $3 \mathrm{~dB}$ linewidth below the resolution of the instrument, $1 \mathrm{~Hz}$. The one to one correspondence represents an optical bandwidth of $9.2 \mathrm{GHz}$. Broader bandwidths could also be achieved at the expense of reduced dynamic range in the comb lines.

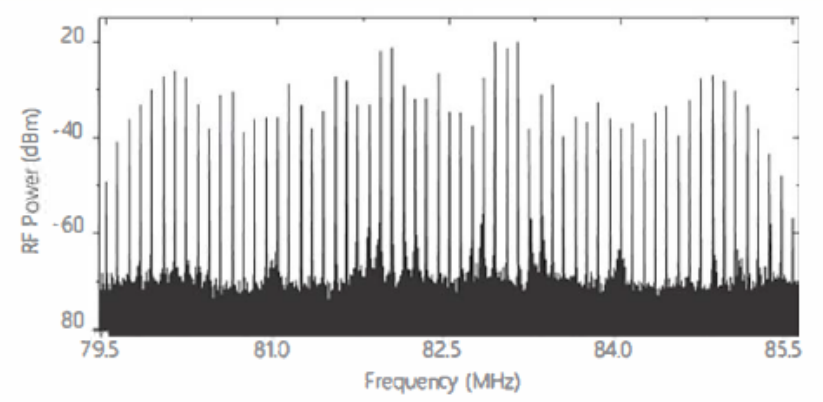

Figure 3. Mlustration of the RF comb generated from the dual comb signal impinged on the photodetector. The central line corresponds to the frequency shift induced by the AOM ( $82.5 \mathrm{MHz})$ and the line spacing to the mismatch between repetition frequencies of the combs $(100 \mathrm{kHz})$. Resolution bandwidth: $0.36 \mathrm{kHz}$.

The process to reach the MIR region of the electromagnetic spectrum is held in a single crystal DFG module whose core is a bulk $40 \mathrm{~mm}$ MgO:PPLN crystal (MOPO1 0.5 40, Covesion Ltd.). DFG is a well established three wave mixing nonlinear process involving three optical signals: customarily designated as pump, signal, and idler. ${ }^{38}$ A pump source at frequency $\omega_{\mathrm{p}}$ drives the process, downconverting the seeded NIR signal combs of frequency $\omega_{S_{1}}=\omega_{S 0} \pm N \times f_{\mathrm{REP}}$ and $\omega_{S 2}=\omega_{S O}+f_{\mathrm{AOM}} \pm N \times$ $\left(f_{\mathrm{REP}}+\Delta\right)$ into MIR idler combs at $\omega_{\mathrm{I1}}=\omega_{\mathrm{P}}-\omega_{\mathrm{S1}}$ and $\omega_{12}=\omega_{\mathrm{P}}$ $-\omega_{S_{2}}\left(\omega_{S_{0}}\right.$ being the emission frequency of the NIR CW laser and $N$ the number of comb lines). Idler combs can be solely generated with proper selection of pump and signal wavelength across the transparency range of the crystal, as PPLN crystals are engineered to be quasi phase matchable (i.e, they can preserve the phase relation between pump and signal across the nonlinear media photons to generate idler radiation) by spatially choosing the poling period of the crystal at a given temperature. ${ }^{39}$

To that end, both signal combs are then amplified with an EDFA delivering an output power of $1.5 \mathrm{~W}$ before combination with $300 \mathrm{~mW}$ of a free nunning pump laser emitting at 1064.25 $\mathrm{nm}$ (QPhotonics, Inc.). Part of the output power of the latter is also split to monitor its emission wavelength with the previous spectrum analyzer. The NIR signal and pump beams are combined in a fiber coupled wavelength division multiplexer (WDM) to be then jointly collimated and focused onto the center of the grating of the crystal with period $29.98 \mu \mathrm{m}$ when the crystal is mounted in a temperature stabilized oven at $140.7^{\circ} \mathrm{C}$. The diameter of the collimator and the focal length of the 
achromatic focusing lens (see Figure 2) is $1.6 \mathrm{~mm}$ and $150 \mathrm{~mm}$, respectively. The MIR idler beam at $3452 \mathrm{~nm}$ is then collimated by a $50 \mathrm{~mm}$ focal length $\mathrm{CaF}_{2}$ lens and filtered out from the residual NIR radiation with a germanium window (cutoff wavelength at $1900 \mathrm{~nm}$ ). Since the setup does not include polarization maintaining components, fiber polarization con trollers are inserted to match the polarization state of the combs and to maximize the conversion efficiency into the crystal, which is attained when the polarization axis of the light is aligned to the thickness of the crystal.

The average experimental conversion efficiency is $73 \mu \mathrm{W} /$ $\mathrm{W}^{2} \mathrm{~cm}$, which is lower than the manufacturer's specifications. ${ }^{40}$ This efficiency is mainly limited by the strong sensitivity of the conversion process to the relative overlap between both input beams. Particularly, for the presented instrument using a single free space arrangement (collimator and lens), the calculated waist radii at the focusing point (assuming Gaussian beams) are 63.7 and $92.4 \mu \mathrm{m}$ for pump and signal, respectively, which translates into focusing parameters $\xi$ of 1.68 and 1.16 (the optimum being $\xi \approx 1.3^{41}$ ). Nonetheless, at the expense of lower conversion efficiency, the employment of optical fiber components and a single crystal arrangement dramatically lessens the complexity of the system, ensuring alignment free coupling of the beams and reducing the free space section to a single pass MIR generation process, as well as featuring improved performance against environmental instabilities, such as temperature related misalignments or mechanical vibrations.

For the above mentioned signal and pump input powers and conversion efficiency, a filtered idler dual comb of $\sim 84 \mu \mathrm{W}$ optical power-induding all optical losses-is generated, corresponding to an average idler power per comb line of $\sim 0.7$ $\mu \mathrm{W}$ in each of the two combs, which is sufficient for high sensitive absorption spectroscopy, as reported in other works of MIR DCS. ${ }^{24}$ The collimated MIR OFCs are then focused on a thermoelectrically cooled MCT photodetector (Vigo Systems, S.A) with an active area of $1 \mathrm{~mm}^{2}$, sensitivity of $82 \mathrm{nW}$ at $3.5 \mu \mathrm{m}$, and $3 \mathrm{~dB}$ cutoff frequency of $\sim 180 \mathrm{MHz}$. Finally, a fast oscilloscope (Rohde \& Schwar, Inc.) is used to sample and acquire the temporal trace from the detector. The RF comb after downconversion from the MIR idler OFCs is also examined, showing no apparent changes in either the distribution of power across the teeth or the linewidth in comparison to the RF comb shown in Figure 3.

\section{RESULTS AND DISCUSSION}

The suitability of the instrument was tested by targeting the absorption feature of methane in the $\mathrm{P}(12)$ manifold of the $\nu 3$ band at $3451.87 \mathrm{~nm}\left(2896.98 \mathrm{~cm}^{1}\right)$. To that end, a $5 \mathrm{~cm}$ single pass cell filled with $1 \% \mathrm{CH}_{4}$ balanced with $\mathrm{N}_{2}$ at 400 Torr at room temperature was placed in the idler beam path during the experimental tests without any additional modification in both electro optic dual comb scheme and DFG module. The gas cell was installed in a custom made flip mount, which allows for easy and swift change between measurement (methane) and reference conditions (in the present experiment, ambient air was used as reference gas) in the idler combs' path.

To fully exploit the speed capabilities of the system, the frequency detuning between combs $(\Delta)$ was increased to 2.5 $\mathrm{MH}_{\mathrm{z}}$ by rapid tuning of the RF electronic settings of the EOM without altering any of the previous features of the RF comb. Therefore, the temporal trace refresh rate between adjacent interferograms is squeezed down to $400 \mathrm{~ns}(1 / \Delta)$. Under these conditions, the time domain signal was synchronously sampled at $5 \mathrm{GS} / \mathrm{s}$, yielding up to $400 \mu \mathrm{s}$ of integration time (or $2 \times 10^{6}$ samples). The Fourier transformation of the set of interfero grams unveils an RF comb whose beatnotes are then processed to extract the information associated with every spectral point, in this case, the intensity (note that the full complex spectrum of the sample cannot be retrieved in the presented configuration with both combs interrogating the sample). This method is employed in order to retrieve the spectral profile of the target absorption feature, as shown in Figure 4, where the ratio between a reference

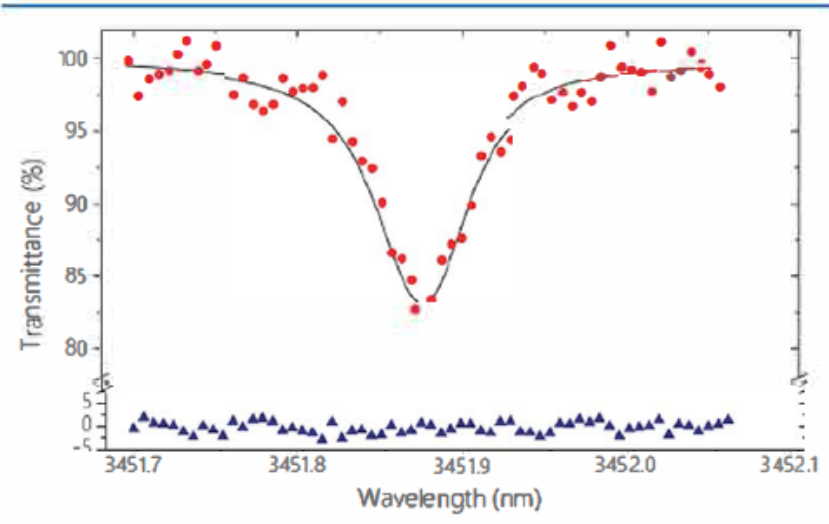

Figure 4. Experimental validation in the $3.5 \mu \mathrm{m}$ region. The optical axis was recovered by upconversion from the RF axis considering the compression factor $f_{\text {REP }} / \Delta$. The red dots represent the trace of the measured transition of methane at $3451.87 \mathrm{~nm}$ in the $\mathrm{P}(12)$ manifold of the $\nu 3$ band for a measurement time of $40 \mu$ s (pressure $=400 \mathrm{Torr}$, temperature $=298 \mathrm{~K}$ ). The solid line shows the spectrum calculated from the HITRAN 2012 database at the same pressure and temperature conditions. The residuals (blue dots) present a standard deviation of $1.23 \%$, showing no systematic deviation.

and a measurement of the gas is depicted. The dots represent the profile containing the amplitude encoded information after an average of 100 interferograms over a total integration time of 40 $\mu$ s. In comparison to the line parameters retrieved from the HITRAN 2012 database $^{42}$ (solid line), the experimental profile shows good agreement with the residuals, revealing a standard deviation of $1.23 \%$.

The signal to noise ratio (SNR) for each comb line is defined as the amplitude of every RF beatnote divided by its standard deviation when no gas sample is placed in the beam path. From this definition, we establish the SNR of the instrument as the average SNR over the whole set of lines of the RF comb. As can be seen in Figure 5, this value scales with the square root of the number of samples (or, similarly, with the integration time). The equivalent average SNR at $1 \mathrm{~s}$ integration time is $8.6 \times 10^{3}$. From this value, the 3 sigma limit of detection (LOD) achievable is estimated as the concentration of methane that yields a signal equal to 3 times the standard deviation of the baseline. The trend of the minimum detectable signal as a function of the integration time is also illustrated in Figure 5. From these values, the equivalent $L O D$ of the instrument at $1 \mathrm{~s}$ and $1 \mathrm{~m}$ absorption path is $1.05 \mathrm{ppm} \times \mathrm{m} \times \mathrm{Hz}^{1 / 2}$ for $\mathrm{CH}_{4}$ at $2896.98 \mathrm{~cm}^{1}$. It is also noteworthy that a quick extrapolation of this result to stronger absorption features of methane (for instance, in the $Q$ branch) leads to a LOD in the region of $\sim 100 \mathrm{ppb} \times \mathrm{m} \times \mathrm{Hz}^{1 / 2}$.

\section{CONCLUSIONS}

MIR DCS is a long sought binomial with tremendous potential for high resolution, real time ultrasensitive molecular spectros copy. To date, a number of promising demonstrations have been 


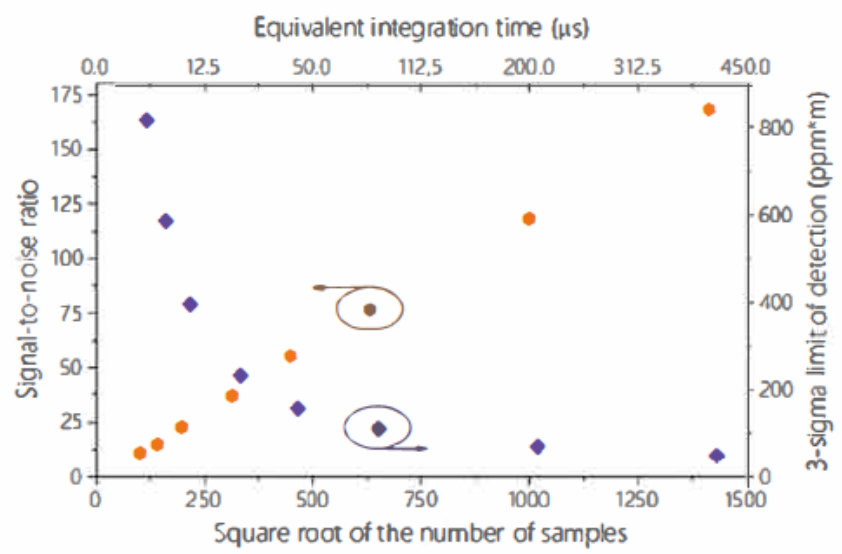

Figure 5. Evolution of the signal to noise ratio and 3 sigma limit of detection (for the target absorption feature) as a function of the integration time. The SNR scales linearty with the number of samples, exhibiting the very high passive mutual coherence of electro optic dual comb a rehitectures.

successfully validated, but adapting the instruments to the specific application still remains challenging, particularly in outside the lab environments. EO DCS platforms are promising candidates to overcome these limitations, as they exhibit rapid tailor made fine tuning of the main optical parameters as well as bottom up capabilities in terms of bandwidth expansion, straightforwand assemblage with the inherent robustness of commercialized components, and inherent mutual coherence between combs. However, most EO DCS molecular spectros copy applicationshave so far been realized in the NIR.region with limited sensitivity in comparison to that achievable in the MIR.

Here, we have presented an instrument that incorporates a simplified single crystal DFG module allowing for the rapid frequency shifting of EO dual combs into the MIR region with adequate power per comb line and intrinsically uncomplicated wavelength tuning capabilities and without sacrificing any of the virtues of the $\mathrm{EO}$ dual comb architecture. Altogether, the presented tool exhibits straightforward acœss to the fingerprint region with complete adaptability to the target spectral feature. Furthermore, since the gas sample under test does not take part in the setup, the system features distinctive robustness to environmental conditions. The performance of the system has been validated by recovering the absorption profile of the molecular transition of methane at $2896.98 \mathrm{~cm}$ ' with high fidelity within tens of microseconds, resulting in an equivalent sensitivity of $\sim 1 \mathrm{ppm} \times \mathrm{m}$ in $1 \mathrm{~s}$. Furthermore, the modular nature of the instrument paves the way for incorporating the range of advancements associated with EO architectures and DFG systems, such as waveguide nonlinear crystals with improved efficiency $\left(\sim 20 \% \mathrm{~W}^{-1} \text { in the case of PPLN) }\right)^{43}$ or new nonlinear crystals with increased transparency deeper in MIR (e.g., orientation patterned GaP/GaAs). ${ }^{44}$

In any respect, the simplicity of the system and the small footprint on which it can be implemented (in this case, all fiber coupled components easily fit in a 19 in. rack module, whereas the free space module covered an area of $250 \mathrm{~cm}^{2}$ ) certainly facilitates its potential deployment for in field operation, thus offering new prospects for the gradual standardization process of lightweight EO dual comb platforms beyond laboratory environ ments. ${ }^{45}$ Especially, we can envision that applications such as monitoring of transient chemical reaction (such as the ones found in processes of combustion) or real time control of gas leaks, for security purposes or environmental studies, may profit from the versatility and ultrafast capabilities of the presented architecture.

\section{AUTHOR INFORMATION}

\section{Corresponding Author}

*E mail: bjerez@ing.uc3m.es. Tel: +3491624 8753.

\section{ORCID}

Borja Jerez: 0000000195430019

Pedro Martín-Mateos: 0000000316569456

\section{Notes}

The authors declare no competing financial interest.

\section{ACKNOWLEDGMENTS}

The authors would like to thank the Spanish Ministry of Economy and Competitiveness for supporting the project under the grant TEC 201452147 R (MOSSI). The work by B.J. has been performed in the frame of an FPU Program, \#FPU014/ 06338, granted by the Spanish Ministry of Education, Culture and Sports. The work by F.W. is supported by the European project H2020 MSCA ITN 2015 (CELTA).

\section{REFERENCES}

(1) Hänsch, T. W. Nobel Lecture: Passion for Precision Rev. Mod. Phys. 2006, 78 (4), 1297-1309.

(2) Newbury, N. R. Searching for Applications with a Fine Tooth Comb. Nat. Photonics 2011, S (4), 186-188.

(3) Coddington, L; Newbury, N.; Swann, W. Dual Comb Spectros copy. Optica 2016, 3 (4), 414-426.

(4) Schiller, S. Spectrometry with Frequency Combs. Opt. Lett. 2002, 27 (9), 766-768.

(5) Schliesser, A.; Picqué, N.; Hänsch, T. W. Mid Infrared Frequency Combs. Nat. Photonics 2012, 6 (7), 440-449.

(6) Galli, I.; Bartalini, S.; Borri, S.; Cancio, P.; Mazzotti, D.; De Natale, P.; Giusfredi, G. Molecular Gas Sensing below Parts per Trillion: Radiocarbon Dioxide Optical Detection. Phys. Rev. Lett 2011, 107(27), 270802.

(7) Keilmann, F.; Gohle, C.; Holzwarth, R. Time Domain Mid Infrared Frequency Comb Spectrometer. Opt. Lett. 2004, 29 (13), $1542-1544$

(8) Schliesser, A.; Brehm, M.; Keilmann, F.; van der Weide, D. Frequency Comb Infrared Spectrometer for Rapid, Remote Chemical Sensing. Opt. Express 2005, 13 (22), 9029-9038.

(9) Bernhardt, B; Sorokin, E.; Jacquet, P; Thon, R; Becker, T.; Sorokina, I. T.; Picqué, N.; Hänsch, T. W. Mid Infrared Dual Comb Spectroscopy with $24 \mathrm{Mm} \mathrm{Cr} 2+: \mathrm{ZnSe}$ Femtosecond Lasers. Appl. Phys. B: Lasers Opt. 2010, 100 (1), 3-8.

(10) Truong, G. W.; Waxman, E. M.; Cossel, K C.; Baumann, E.; Klose, A.; Giorgetta, F. R; Swann, W. C.; Newbury, N. R; Coddington, L Accurate Frequency Referencing for Fieldable Dual Comb Spectros copy. Opt. Express 2016, 24 (26), 30495-30504.

(11) Schroeder, P. J.; Wright, R J.; Coburn, S.; Sodergren, B.; Cossel, K. C.; Droste, S.; Truong, G. W.; Baumann, E.; Giorgetta, F. R; Coddington, I; Newbury, N. R; Rieker, G. B. Dual Frequency Comb Iaser Absorption Spectroscopy in a $16 \mathrm{MW}$ Gas Turbine Exhaust. Proc. Combust. Inst. 2017, 36 (3), 4565-4573.

(12) Cossel, K C.; Waxman, E. M.; Giorgetta, F. R.; Cermak, M.; Coddington, I. R; Hesselius, D.; Ruben, S.; Swann, W. C.; Truong, G. W.; Rieker, G. B.; Newbury, N. R. Open Path Dual Comb Spectroscopy to an Airbome Retroreflector. Optica 2017, 4 (7), 724-728.

(13) Coburn, S.; Alden, C. B.; Wright, R. J.; Cossel, K; Baumann, E.; Truong, G. W; Giongetta, F. R; Sweeney, C.; Newbury, N. R; Prasad, K; Coddington, I.; Rieker, G. B. Regional Trace Gas Source Attribution Using a Field Deployed Dual Frequency Comb Spectrometer. Optica 2018, 5 (4), 320-327. 
(14) Villares, G.; Hugi, A.; Blaser, S.; Faist, J. Dual Comb Spectroscopy Based on Quantum Cascade Laser Frequency Combs. Nat. Commun. 2014, 5, 5192 .

(15) Westherg, J.; Sterczewski, L. A.; Wysodki, G. Mid Infrared Multiheterodyne Spectroscopy with Phase Locked Quantum Cascade Iasers. Appl. Phys. Lett. 2017, 110 (14), 141108.

(16) Sterczewski, L. A.;Westberg, J.; Patrick, C. L.; Kim, C. S.; Kim, M.; Canedy, C. L; Bewley, W. W.; Merritt, C. D.; Vurgaftman, L; Meyer, J. R; Wysodi, G. Multiheterodyne Spectroscopy Using Interband Cascade Lasers. Opt. Eng. 2018, 57 (1), 11014.

(17) Yu, M.; Okawachi, Y.; Griffith, A. G.; Picqué, N.; Lipson, M.; Gaeta, A. L. Silicon Chip Based Mid InfraredDual Comb Spectroscopy (arXiv:1610.01121).

(18) Zhang, Z.; Gardiner, T.; Reid, D. T. Mid Infrared Dual Comb Spectroscopy with an Optical Parametric Oscillator. Opt. Iett. 2013, 38 (16), 3148-3150.

(19) Jin, Y.; Cristescu, S. M.; Harren, F. J. M.; Mandon, J. Two Crystal Mid Infrared Optical Parametric Oscillator for Absorption and Dispersion Dual Comb Spectroscopy. Opt. Lett. 2014, 39 (11), 3270-3273.

(20) Jin, Y.; Cristescu, S. M.; Harren, F. J. M.; Mandon, J. Femtosecond Optical Parametric Oscillators toward Real Time Dual Comb Spec troscopy. Appl. Phys. B: Lasers Opt. 2015, 119 (1), 65-74.

(21) Baumann, E.; Giorgetta, F. R; Swann, W. C.; Zolot, A. M.; Coddington, I; Newbury, N. R. Spectroscopy of the Methane $\nu 3$ Band with an Accurate Midinfrared Coherent Dual Comb Spectrometer. Phys. Rev. A: At, Mol, Opt. Phys. 201 1, 84 (6), 62513.

(22) Zhu, F.; Bicer, A.; Askar, R; Bounds, J.; Kolomenskii, A. A.; Kelessides, V.; Amani, M.; Schuessler, H. A. Mid Infrared Dual Frequency Comb Spectroscopy Based on Fiber Lasers for the Detection of Methane in Ambient Air. Iaser Phys. Lett 2015, 12 (9), 95701.

(23) Ycas, G.; Giorgetta, F. R ; Baumann, E.; Coddington, L; Herman, D.; Diddams, S. A; Newbury, N. R. High Coherence Mid Infrared Dual Comb Spectroscopy Spanning 2.6 to $5.2 \mu \mathrm{m}$. Nat. Photonics 2018, 12, 202-208.

(24) Yan, M.; Luo, P. L.; Iwakuni, K; Millot, G.; Hänsch, T. W.; Picqué, N. Mid Infrared Dual Comb Spectroscopy with Electro Optic Modulators. Light: Sci. Appl. 2017, 6 (10), e 17076.

(25) Coddington, I; Swann, W. C; Newbury, N. R. Coherent Multiheterodyne Spectroscopy Using Stabilized Optical Frequency Combs. Phys. Rev. Lett. 2008, 100 (1), 13902.

(26) Zolot, A. M.; Giorgetta, F. R; Baumann, E.; Nicholson, J. W.; Swann, W. C.; Coddington, L; Newbury, N. R. Direct Comb Molecular Spectroscopy with Accurate, Resolved Comb Teeth over $43 \mathrm{THz}$. Opt. Lett. 2012, 37 (4), 638-640.

(27) Rieker, G. B.; Giorgetta, F. R; Swann, W. C.; Kofler, J.; Zolot, A. M.; Sinclair, L. C.; Baumann, E.; Cromer, C; Petron, G.; Sweeney, C.; Tans, P. P.; Coddington, L; Newbury, N. R. Frequency Comb Based Remote Sensing of Greenhouse Gases over Kilometer Air Paths. Optica 2014, 1 (5), 290.

(28) Long, D. A.; Fleisher, A. J.; Douglass, K. O.; Maxwell, S. E.; Bielska, K.; Hodges, J. T.; Plusquellic, D. F. Multiheterodyne Spectroscopy with Optical Frequency Combs Generated from a Continuous Wave Iaser. Opt. Lett. 2014, 39 (9), 2688-2690.

(29) Martin Mateos, P.; Ruiz Llata, M.; Posada Roman, J.; Acedo, P. Dual Comb Architecture for Fast Spectroscopic Measurements and Spectral Characterization. IEEE Photonics Technol. Lett. 2015, 27 (12), 1309-1312.

(30) Martin Mateos, P.; Jerez, B; Acedo, P. Dual Electro Optic Optical Frequency Combs for Multiheterodyne Molecular Dispersion Spec troscopy. Opt. Express 2015, 23 (16), 21149-21158.

(31) Fleisher, A. J.; Bjork, B. J.; Bui, T. Q.; Cossel, K. C.; Okumura, M.; Ye, J. Mid Infrared Time Resolved Frequency Comb Spectroscopy of Transient Free Radicals. J. Phys. Chem. Lett. 2014, 5 (13), 2241-2246.

(32) Jerez, B.; Martin Mateos, P.; Prior, E.; de Dios, C.; Acedo, P. Dual Optical Frequency Comb Architecture with Capabilities from Visible to Mid Infrared. Opt. Express 2016, 24 (13), 14986-14994.
(33) Millot, G.; Pitois, S.; Yan, M.; Hovhannisyan, T.; Bendahmane, A.; Hänsch, T. W.; Picqué, N. Frequency Agjle Dual Comb Spectroscopy. Nat. Photonics 2016, 10, 27-30.

(34) Fleisher, A. J.; Long, D. A.; Reed, Z. D.; Hodges, J. T.; Plusquellic, D. F. Coherent Cavity Enhanced Dual Comb Spectroscopy. Opt. Express 2016, 24 (10), 10424-10434.

(35) Durán, V.; Andrekson, P. A; Torres Company, V. Electro Optic Dual Comb Interferometry over $40 \mathrm{~nm}$ Bandwidth. Opt. Lett. 2016, 41 (18), 4190-4193.

(36) Wang, S; Fan, X; Xu, B.; He, Z. Dense Electro Optic Frequency Comb Generated by Two Stage Modulation for Dual Comb Spectros copy. Opt. Iett. 2017, 42 (19), 3984-3987.

(37) Martín Mateos, P.; Porno, A; Acedo, P. Fully Adaptable Electro Optic Dual Comb Generation. IEEE Photonics Technol. Lett. 2018, 30 (2), 161-164

(38) Armstrong, J. A.; Bloembergen, N; Ducuing, J.; Pershan, P. S. Interactions between Light Waves in a Nonlinear Dielectric. Phys. Rev. 1962,127 (6), 1918-1939.

(39) Hum, D. S.; Fejer, M. M. Quasi Phasematching. C. R. Phys. 2007, 8 (2), 180-198.

(40) Richter, D.; Weibring, P. Ultra High Precision Mid IR Spectrometer I: Design and Analysis of an Optical Fiber Pumped Difference Frequency Generation Source. Appl. Phys. B: Lasers Opt 2006, 82 (3), 479-486.

(41) Chen, W.; Cousin, J.; Poullet, E.; Burie, J.; Boucher, D.; Gao, X; Sigrist, M. W.; Tittel, F. Continuous Wave Mid Infrared Laser Sources Based on Difference Frequency Generation. C. R Phys. 2007, 8, 11291150.

(42) Rothman, L. S.; Gordon, I. E.; Babikov, Y.; Barbe, A.; Chris Benner, D.; Bernath, P.F.; Birk, M.; Bizzocchi, L.; Boudon, V.; Brown, L. R; Campargue, A.; Chance, K; Cohen, E. A.; Coudert, L. H.; Devi, V. M.; Drouin, B. J.; Fayt, A.; Flaud, J. M.; Gamache, R. R; Harrison, J. J.; Hartmann, J. M.; Hill, C.; Hodges, J. T.; Jacquemart, D.; Jolly, A.; Lamouroux, J.; Le Roy, R J.; Li, G.; Long, D. A.; Lyulin, O.M; Madkie, C. J.; Massie, S. T.; Mikhailenko, S.; Müller, H. S. P.; Naumenko, O. V.; Nikitin, A. V.; Orphal, J.; Perevalov, V.; Perrin, A.; Polovtseva, E. R; Richard, C.; Smith, M. A. H.; Starikova, E.; Sung, K; Tashkun, S.; Tennyson, J.; Toon, G. C.; Tyuterev, V. G.; Wagner, G. The HITRAN2012 Molecular Spectroscopic Database. J. Quant. Spectrosc. Radiat. Transfer 2013, 130, 4-50.

(43) Krzempek, K; Abramski, K. M.; Nikodem, M. All Fiber Mid Infrared Difference Frequency Generation Source and Its Application to Molecular Dispersion Spectroscopy. Laser Phys. Lett 2017, 14 (9), 95702.

(44) Kara, O.; Maidment, L.; Gardiner, T.; Schunemann, P. G.; Reid, D. T. Dual Comb Spectroscopy in the Spectral Fingerprint Region Using OPGaP Optical Parametric Oscillators. Opt. Express 2017, 25 (26), 32713-32721.

(45) Posada Roman, J. E.; Angelina, H.; Jerez, B.; Ruiz Llata, M.; Acedo, P. Laser Range Finder Approach Based on a Fieldable Electro Optic Dual Optical Frequency Comb: A Proof of Concept. Appl. Opt. 2017, 56 (22), 6087. 